\title{
Analysis of PET Parameters Predicting Response to Radiotherapy for Myeloid Sarcoma
}

\author{
Kyu Hye Choi
}

Seoul Saint Mary's Hospital

Jin Ho Song

Seoul Saint Mary's Hospital

\section{Yoo-Kang Kwak}

Catholic University of Korea Incheon Saint Mary's Hospital

\section{Eun Young Park}

Catholic University of Korea Incheon Saint Mary's Hospital

Jong Hoon Lee

Catholic University of Korea Saint Vincent's Hospital

HongSeok Jang ( $\nabla$ hsjang11@catholic.ac.kr)

Seoul St Mary's Hospital https://orcid.org/0000-0003-0326-272X

\section{Research}

Keywords: myeloid sarcoma, chloroma, radiotherapy, SUVmax, metabolic response

Posted Date: November 24th, 2020

DOl: https://doi.org/10.21203/rs.3.rs-113048/v1

License: (c) (i) This work is licensed under a Creative Commons Attribution 4.0 International License. Read Full License

Version of Record: A version of this preprint was published at PLOS ONE on December 20th, 2021. See the published version at https://doi.org/10.1371/journal.pone.0261550. 


\section{Abstract}

Background: Positron-emission tomography (PET)-CT has recently been used for diagnostic imaging and radiotherapy for myeloid sarcoma, but there is little research on predicting the response of radiotherapy. The aim of this study was to analyze the association between PET-CT variables and the response to radiotherapy in patients with myeloid sarcoma.

Methods: This study was conducted in myeloid sarcoma patients who received radiotherapy and PET-CT before and after radiotherapy. The response to radiotherapy was evaluated based on the European Organization for Research and Treatment of Cancer PET response criteria, and binary regression analysis was performed to assess the factors predicting reductions in the SUVmax.

Results: Twenty-seven sites in 12 patients were included in the study. The 27 irradiated sites included 14 soft tissues, 11 bones, and two organs. Complete metabolic responses were seen in 24 patients after radiotherapy, a partial metabolic response in one, and progressive metabolic disease in two patients. The prescribed dose of more than $3000 \mathrm{cGy}_{10}$ was significantly greater in the treatment control group $(P=$ 0.024). In binary logistic regression analysis predicting reductions in the SUVmax of more than $70 \%$ after radiotherapy, the pretreatment SUVmax $(\geq 7.5)$ and further chemotherapy after radiotherapy showed significant differences in univariate and multivariate analyses.

Conclusions: Good metabolic responses (complete or partial) to radiotherapy were achieved in $92.6 \%$ of the myeloid sarcoma patients. Radiation doses $<3000 \mathrm{cGy}_{10}$ and increased SUVmax were related to treatment failure and high SUVmax before radiotherapy was a factor influencing SUVmax reduction. Further large-scale studies are needed.

\section{Background}

Myeloid sarcoma is a solid tumor formed by leukemic cells outside the bone marrow and was also called chloroma in the 19th century because the tumors showed a greenish hue from the myeloperoxidase enzyme [1]. It usually occurs in patients with acute myeloid leukemia (AML) and other myeloproliferative diseases or myelodysplastic conditions, and rarely, in lymphoid leukemia. Tumors usually appear in bones, but can occur in any part of the body including the skin, and are often found in imaging studies such as computed tomography (CT) or magnetic resonance imaging (MRI) without symptoms in approximately $50 \%$ of the patients [2]. Patients with leukemia and myeloid sarcoma have a poor prognosis, so it is recommended that local treatment be actively performed $[3,4]$.

Although myeloid sarcoma can be diagnosed through CT or MRI, tumor often invades the skin or connective tissue. For this, rather than anatomical imaging, functional imaging tests such as positron emission tomography (PET)-CT has recently been used to examine the sites that cannot be evaluated with conventional imaging and the extent of lesion involvement [5]. Among the local treatments, radiation therapy is known to have a relatively good treatment response and symptom relief rate [6, 7]. In radiotherapy planning, PET-CT helps to delineate the targets of radiotherapy and can be used to assess 
the response to therapy [8]. However, little is known about the usefulness of PET-CT in the treatment of myeloid sarcoma, and no research has been conducted on whether it can be used to predict the outcome of radiotherapy.

The purpose of this study was to analyze the relationship between the parameters of PET-CT and the treatment response of myeloid sarcoma patients treated with radiotherapy and identify the potential outcome predictors of radiation therapy.

\section{Materials And Methods}

This study was a retrospective study of patients who received radiation therapy for myeloid sarcoma and PET-CT before and after treatment at Seoul St. Mary's Hospital between March 2015 and August 2019. Prior to treatment, the patient's diagnosis, previous bone marrow transplantation (BMT), and total body irradiation (TBI) history were reviewed through the medical records.

PET-CT was performed before radiotherapy, and the maximum standardized uptake value (SUVmax) before treatment and 3-6 months after the completion of radiotherapy were extracted and the difference was converted into a percentage. For radiotherapy, the target was delineated through PET-CT, then 5$10 \mathrm{~mm}$ was extended from the gross tumor volume (GTV) to the planning target volume (PTV). Both 3dimensional conformal radiotherapy (3D-CRT) and intensity-modulated radiotherapy (IMRT) were included as radiotherapy techniques and the biologically effective dose (BED) was calculated for each case using an alpha/beta ratio of 10 to compare different dose schedules.

The criteria for the response to PET were set based on the European Organization for Research and Treatment of Cancer (EORTC) PET response criteria [9]. For analysis of the treatment dose for radiation therapy, the radiation dose converted to BED with an alpha/beta ratio of 10 (BED10) and the volume of the lesion before treatment were extracted. Recurrence was defined as new lesion development during the follow-up period after the response to radiation therapy. The pattern of recurrence was investigated through imaging studies every 3-6 months after treatment. In the case of recurrence, the recurrence pattern was described in detail. The recurrence patterns were defined as follows. An in-field recurrence was when $95 \%$ of the recurrence volume was within the $90 \%$ isodose curve of the radiotherapy field, and a marginal recurrence was assigned when $20-95 \%$ of the recurrence volume was within the $90 \%$ isodose curve.

The chi-squared test was used to analyze the characteristics of the two groups according to the treatment response, and binary logistic regression analysis was performed to analyze the factors predicting a large SUVmax change. SPSS for Windows, version 24 (IBM Corp Armonk, NY, USA) was used for the statistical analyses. This study was approved by the Institutional Review Board (IRB) of Seoul St. Mary's Hospital (IRB No. KC20RISI0365). The requirement for informed consent was waived by the IRB due to the retrospective nature of the study. 


\section{Results}

A total of 135 lesions were irradiated during the investigation period, and 27 sites in 12 patients who underwent PET-CT before and after treatment were included in the analysis. Patients with AML, acute lymphoblastic leukemia ( $A L L)$, and chronic myelocytic leukemia $(C M L)$ were included, of which eight received BMT before radiation therapy and four of them received TBI. Most of the patients had uncontrolled leukemia, and seven died during the follow-up period, of which five were recorded as related to infections. Table 1 lists the characteristics of the 12 patients. In the 27 irradiated sites (Table 2), soft tissue was the most common (14 patients, $51.9 \%$ ), followed by bone (11 patients, $40.7 \%$ ), and organs ( 2 patients, $7.4 \%)$. 
Table 1

Patient characteristics $(n=12)$

\begin{tabular}{lllr} 
Characteristic & N & \% \\
\hline Sex & & & 66.7 \\
\hline & Male & 8 & 33.3 \\
& Female & 4 & \\
\hline Age & & Median $37.5(14-52)$ & 83.3 \\
& Adult & 10 & 16.7
\end{tabular}

Disease

AML

ALL

CML

Previous BMT

No

Yes

Previous TBI

No

4

Yes

Not-BMT

TBI dose

1200 cGy/ 6 fractions

1320 cGy/8 fractions

1

3

4

4

4

8

5

6

1

33.3

66.7

66.7

Leukemia controlled

No

10

Yes

2

Survival

Survival

5

Death

7
83.3

16.7

41.7

33.3

33.3

33.3

8.3

25.0

58.3

Cause of death 


\begin{tabular}{|cccc|}
\hline Characteristic & N & $\%$ \\
\hline Infection & 5 & 41.7 \\
\hline Disease progression & 1 & 8.3 \\
\hline Treatment-related complication & 1 & 8.3 \\
\hline $\begin{array}{l}\text { AML, acute myeloid leukemia; ALL, acute lymphoblastic leukemia; CML, chronic myelocytic leukemia; } \\
\text { BMT, bone marrow transplantation; TBI; total body irradiation. }\end{array}$ & \\
\hline
\end{tabular}


Table 2

Characteristics of the irradiated sites $(n=27)$

\begin{tabular}{lllr} 
Characteristic & N & $\%$ \\
\hline Site & & & \\
& Soft tissue & 14 & 51.9 \\
& Bone & 11 & 40.7 \\
& Organ & 2 & 7.4
\end{tabular}

Disease

ALL

12

44.4

AML

13

48.1

CML

2

7.4

RT volume

Median 43.06 (0.26-486.53)

$<40 \mathrm{~cm}^{3}$

13

48.1

$\geq 40 \mathrm{~cm}^{3}$

14

51.9

PreRT SUVmax

Median 7.47 (1.81-21.38)

$<7.5$

14

51.9

$\geq 7.5$

13

48.1

RT technique

3D-CRT

IMRT

RT fraction size

200 cGy/fraction

$250 \mathrm{cGy} /$ fraction

$300 \mathrm{cGy} /$ fraction

$500 \mathrm{cGy} /$ fraction

BED10

$<3000$ cGy $_{10}$

$\geq 3000 \mathrm{cGy}_{10}$
9

18

33.3

66.7

22.2

25.9

29.6

22.2

Median 3125 (2400-5000)

12

44.4

15 
$A M L$, acute myeloid leukemia; $A L L$, acute lymphoblastic leukemia; $C M L$, chronic myelocytic leukemia; RT, radiotherapy; SUVmax, maximum standardized uptake value; 3D-CRT, 3-dimensional conformal radiotherapy; IMRT, intensity-modulated radiotherapy; BED10, biologically effective dose (alpha/beta ratio $=10)$.

The SUVmax in PET-CT before radiotherapy was in the range of 1.81-21.38, with a median of 7.47, and the patients were classified into high and low SUVmax groups based on an SUVmax of 7.5. The radiation treatment dose was 200-500 cGy per fraction, and when converted to BED10, the median value was $3125 \mathrm{cGy}_{10}$, ranging from $2400-5000 \mathrm{cGy}_{10}$. The patients were classified into high and low irradiation groups based on a median BED10 of 3000 .

Evaluation of the response to PET-CT was performed at a median of 3.5 months (range, 1.9-7.3). The PET response after radiotherapy was classified as complete metabolic remission (CMR) at 24 sites (88.9\%), partial metabolic remission (PMR) at one site (3.7\%), and progressive metabolic disease (PMD) at two sites (7.4\%). The change in SUVmax ranged from a decrease of $88.0 \%(-88.0 \%)$ to an increase of $263.0 \%(+263.0 \%)$, showing a median change of $-70.9 \%$. Figure 1 shows a waterfall plot of the maximum change in the SUVmax. The two sites showing PMD were both arm muscles, and the pretreatment SUVmax values were 2.35 and 1.81, respectively (Table 3). In one ALL patient with extramedullary relapse, the follow-up SUVmax values of two irradiated lesions in PET-CT 6.4 months after completion of radiotherapy increased to 6.66 and 6.57 , respectively. There was no PET response, but the patient's symptoms improved. PET achieved CMR with additional chemotherapy, but the patient died from an infection related to chemotherapy. 
Table 3

Characteristics of progressive metabolic lesions after radiotherapy

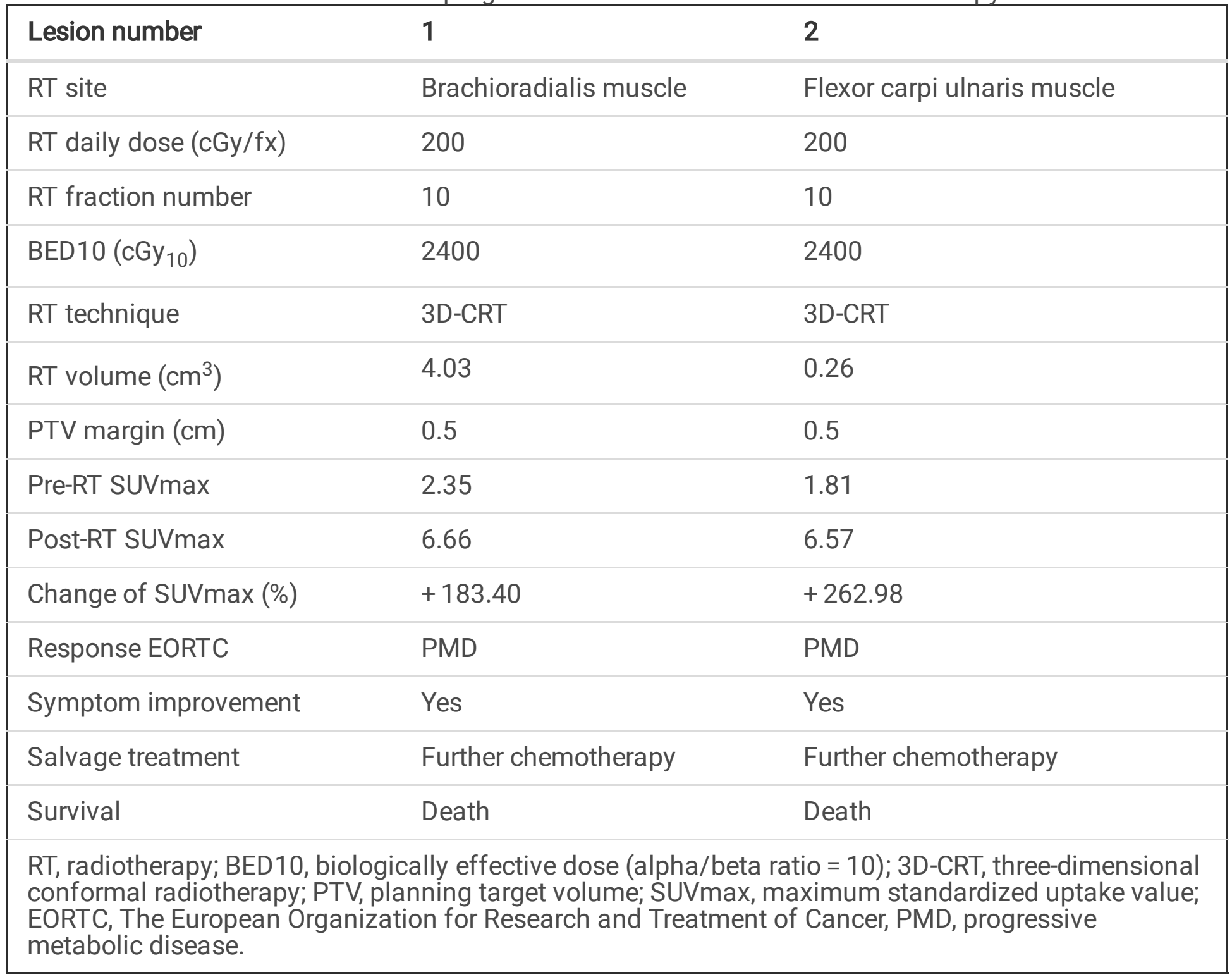

During the follow-up period, there were recurrences in three sites, two sites with in-field recurrences and one site with marginal recurrence. The characteristics of the relapsed lesions are summarized in Table 4. The EORTC PET responses to radiotherapy were CMR in all three lesions and the decreases in the SUVmax after treatment ranged from 27.84-84.12. The relapse periods were 18.6, 8.7, and 6.7 months after the completion of radiotherapy. After recurrence, radiation therapy with a salvage aim was performed again, and the lesions were all controlled. 
Table 4

Characteristics of recurrent lesions after radiotherapy

\begin{tabular}{|c|c|c|c|}
\hline Lesion number & 8 & 15 & 21 \\
\hline Diagnosis & AML & ALL & ALL \\
\hline Age at diagnosis & 52 & 49 & 14 \\
\hline Sex & Female & Female & Male \\
\hline Previous BMT history & Yes & Yes & No \\
\hline RT site & Left lower extremity & Abdominal wall & Lumbar spine \\
\hline RT daily dose (cGy/fx) & 300 & 250 & 200 \\
\hline RT fraction number & 10 & 10 & 10 \\
\hline BED10 $\left(\mathrm{cGy}_{10}\right)$ & 3900 & 3125 & 2400 \\
\hline RT technique & IMRT & IMRT & 3D-CRT \\
\hline RT volume $\left(\mathrm{cm}^{3}\right)$ & 17.39 & 123.32 & 3.77 \\
\hline PTV margin (cm) & 0.5 & 1 & - \\
\hline Pre-RT SUVmax & 5.46 & 14.74 & 6.06 \\
\hline Post-RT SUVmax & 3.94 & 2.34 & 3.26 \\
\hline Change of SUVmax (\%) & -27.84 & -84.12 & -46.20 \\
\hline Response EORTC & CMR & CMR & CMR \\
\hline Relapse period (months) & 18.6 & 8.7 & 6.7 \\
\hline Pattern of recurrence & In-field & Marginal & In-field \\
\hline Systemic disease at recurrence & Uncontrolled & Uncontrolled & Controlled \\
\hline Survival & Survival & Survival & Survival \\
\hline Salvage treatment & re-RT & RT & re-RT \\
\hline \multicolumn{4}{|c|}{$\begin{array}{l}\text { AML, acute myeloid leukemia; ALL, acute lymphocytic leukemia; RT, radiotherapy; BED10, biologically } \\
\text { effective dose (alpha/beta ratio = 10); IMRT, intensity-modulated radiotherapy; 3D-CRT, three- } \\
\text { dimensional conformal radiotherapy; PTV, planning target volume; SUVmax, maximum standardized } \\
\text { uptake value; EORTC, The European Organization for Research and Treatment of Cancer; CMR, } \\
\text { complete metabolic response; re-RT, re-irradiation. }\end{array}$} \\
\hline
\end{tabular}

The two lesions with disease progression and the three lesions with recurrence were defined as a treatment failure group, and the clinical factors were compared with the controlled groups and analyzed (Table 5). There was no significant difference in the radiation volume, site, and SUVmax before treatment, but the SUVmax after treatment showed a value of 2 or more in the treatment failure group, with a marginal statistical difference $(P=0.057)$. Although there was no significant difference between the 
radiation technique and the dose per fraction, the number of lesions irradiated with more than $3000 \mathrm{cGy}_{10}$ based on BED10 was significantly greater in the treatment control group $(P=0.024)$.

Table 5

Comparison of clinical factors between treatment failure and controlled groups

\section{Characteristic}

$\begin{array}{ll}\text { Treatment failure group } & \text { Treatment controlled group } P \text {-value } \\ (n=5) & (n=22)\end{array}$

Site

0.296

Bone

Non-bone

RT volume

$$
\begin{aligned}
& <40 \mathrm{~cm}^{3} \\
& \geq 40 \mathrm{~cm}^{3}
\end{aligned}
$$

PreRT SUVmax

$$
\begin{aligned}
& <7.5 \\
& \geq 7.5
\end{aligned}
$$

PostRT SUVmax

$\begin{array}{lll}<2 & 0 & 10 \\ \geq 2 & 5 & 12\end{array}$

$\mathrm{RT}$ technique

$\begin{array}{lll}\text { 3D-CRT } & 3 & 6 \\ \text { IMRT } & 2 & 16\end{array}$

RT fraction size

4
10
1

\section{0}

12

0.114

9

13

0.163

0

12

0.057

10

2

0.161

6

16

0.185

$\begin{array}{lll}200-300 \text { cGy/fraction } & 5 & 16 \\ 500 \mathrm{cGy} / \text { fraction } & 0 & 6\end{array}$

BED10

$\begin{array}{lll}<3000 \mathrm{cGy}_{10} & 3 & 3 \\ \geq 3000 \mathrm{cGy}_{10} & 2 & 19\end{array}$

RT, radiotherapy; SUVmax, maximum standardized uptake value; 3D-CRT, three-dimensional conformal radiotherapy; IMRT, intensity-modulated radiotherapy; BED10, biologically effective dose $($ alpha/beta ratio $=10)$. 
In this study, the median change in the SUVmax was - 70\%, and binary logistic regression analysis was performed to predict the changes in the SUVmax. In the univariate analysis, a high SUVmax of 7.5 or more before radiotherapy $(P=0.017)$ and further chemotherapy after radiotherapy $(P=0.018)$ were found to be significant factors. In the multivariate analysis, both of these factors were significant (further chemotherapy after radiotherapy, $P=0.026$; pretreatment SUVmax, $P=0.027)$. Changes in the SUVmax decreased by more than $70 \%$ in cases when chemotherapy was not administered after radiotherapy and the SUVmax before treatment was higher than 7.5. The results are described in Table 6.

Table 6

Binary logistic regression analysis predicting reductions in SUVmax of $\geq 70 \%$ after radiotherapy

\begin{tabular}{|c|c|c|c|c|}
\hline \multirow[t]{2}{*}{ Characteristic } & \multirow{2}{*}{$\begin{array}{l}\text { Univariate } \\
P \text {-value }\end{array}$} & \multicolumn{3}{|c|}{ Multivariate analysis } \\
\hline & & Odds ratio & $95 \% \mathrm{Cl}$ & $P$-value \\
\hline RT site (Non-bone) & 0.816 & & & \\
\hline RT volume $\left(40 \mathrm{~cm}^{3}\right)$ & 0.335 & & & \\
\hline Further chemotherapy after RT (yes) & 0.018 & 0.062 & $0.005-0.718$ & 0.026 \\
\hline Pretreatment SUVmax ( $\geq 7.5$ ) & 0.017 & 13.862 & $1.350-142.341$ & 0.027 \\
\hline RT dose $\left(\right.$ BED10) $\left(\geq 3000 \mathrm{cGy}_{10}\right)$ & 0.092 & & & \\
\hline
\end{tabular}

\section{Discussion}

Treatment for myeloid sarcoma-related leukemia is typically sensitive to chemotherapy and long-term remission has been reported when allogeneic hematopoietic stem cell transplantation was performed [10, 11]. Local treatment may be considered for organ invasion causing symptoms that may be lifethreatening, such as to the spinal cord [5]. However, the risk of leukemia progression is high and the survival rate is not affected by local treatment alone [12]. Thus, radiation therapy is recommended for palliative purposes in combination with other treatments for symptomatic isolated lesions or patients who underwent previous transplantations [13].

Previous studies reported that the local recurrence of the treated lesion was low when radiotherapy was performed as a local treatment. Bakst et al. reported a $97 \%$ symptom relief effect for tumors irradiated with at least 20 Gy and showed a higher complete response rate compared to patients who did not receive radiation therapy [6]. Song et al. reported a symptomatic response of $85.7 \%$ when lesions were irradiated at $20 \mathrm{~Gy}$ with $2 \mathrm{~Gy}$, and that small lesions less than $6 \mathrm{~cm}$ and soft tissue showed good complete remission rates [7]. In the present study, we evaluated the response of PET-CT according to EORTC PET criteria. 
After radiotherapy, the SUVmax before and after treatment achieved CMR at 24 sites (88.9\%) and PMR at one site (3.7\%). In previous studies, PET-CT was reported to be useful as a diagnostic tool for extramedullary disease in patients with leukemia. In a recent prospective study, PET-CT had $77 \%$ sensitivity and $97 \%$ specificity for detecting extramedullary disease in AML patients [14]. In the present study, although the correlation between the SUVmax response and recurrence was difficult to analyze due to the lack of recurrence cases and the number of samples, we analyzed the factors affecting the recurrence and PMD showing treatment failure. In the results, treatment failures tended to be higher when the BED was low and the SUVmax increased after treatment. The results showed that the possibility of treatment control may be high when BED10 is irradiated with more than $3000 \mathrm{cGy}_{10}$. Bakst et al. [6] proposed a schedule of $24 \mathrm{~Gy}$ in 12 fractions, which is BED10 $2880 \mathrm{cGy}_{10}$, suggesting that the higher dose in the current study could help control the PET response and recurrence.

Factors influencing the degree of reduction in the SUVmax were also analyzed in this study. A high SUVmax before treatment was identified as a factor influencing reductions in the SUVmax. This study differed from previous studies in that the degree of the decrease in the SUVmax after radiotherapy for myeloid sarcoma was analyzed. The degree of the decrease in the SUVmax was not related to the radiation treatment site, the irradiated volume, or the radiation dose. When reviewing the description of the three recurrent sites, the radiation treatment site, volume, radiation dose, and SUVmax before treatment showed different characteristics, and additional research is warranted. Further large-scale studies are needed to determine whether changes in the SUVmax are related to the recurrence or survival of myeloid sarcoma patients after radiotherapy. In addition, the radiation therapy doses were diversely distributed due to the limitations of the retrospective study. The BED10 showed a somewhat higher tendency as the radiation volume increased in the linear correlation analysis $(P=0.044)$, but a weak linear relationship $(r=0.391)$. The scatter plot (Fig. 2) showed the heterogeneity of BED10, suggesting that a consensus of the schedule for the radiation treatment dose is necessary.

In conclusion, radiotherapy is a non-invasive local treatment modality that achieves good local control and metabolic response in patients with myeloid sarcoma, and a high SUVmax value before treatment was associated with decreases in the SUVmax. The use of PET-CT before and after treatment is expected to be a useful tool for evaluating the response to treatment in addition to diagnostic purposes, and additional studies on PET-CT as an imaging test for treatment response and prognosis should be performed.

\section{Abbreviations}

PET: Positron-emission tomography; AML: Acute myeloid leukemia; CT: Computed tomography; MRI: Magnetic resonance imaging; BMT: Bone marrow transplantation; TBI: Total body irradiation; SUVmax: Maximum standardized uptake value; GTV: Gross tumor volume; PTV: Planning target volume; 3D-CRT: 3dimensional conformal radiotherapy; IMRT: Intensity-modulated radiotherapy; BED: Biologically effective dose; EORTC: European Organization for Research and Treatment of Cancer; BED10: BED with an alpha/beta ratio of 10; IRB: Institutional Review Board; ALL: Acute lymphoblastic leukemia; CML: Chronic 
myelocytic leukemia; CMR: Complete metabolic remission; PMR: Partial metabolic remission; PMD: Progressive metabolic disease

\section{Declarations}

\section{Ethical Approval and Consent to Participate}

This study was approved by the Institutional Review Board of the Korea Association of Health Promotion (approval No. KC20RISI0365). Informed consent was waived due to the retrospective study design.

\section{Consent for publication}

All authors have given consent for publication.

\section{Availability of supporting data}

The dataset used and analyzed during the current study are available from the corresponding author on reasonable request.

\section{Competing interests}

All authors declare that they have no conflict of interest.

\section{Funding}

The study did not receive specific grants from public or commercial funding agencies.

\section{Authors' Contributions}

All of the authors participated in designing this study. $\mathrm{KHC}$, JHS, and HSJ performed the data collection. $\mathrm{KHC}$, JHS, YKK, and EYP undertook the statistical analyses. KHC, JHS, JHL, and HSJ analyzed and interpreted the data. KHC and JHS wrote the first draft of the manuscript, which was reviewed by all of the other authors, who also provided further contributions and suggestions.

\section{Acknowledgement}

We would like to thank the participants who made this study possible. 


\section{References}

1. King C: A Case of Chloroma with Orbital Involvement Locally Benefited by X-Ray Therapy. Trans Am Ophthalmol Soc 1934, 32:340-353.

2. Singh $\mathrm{A}$, Kumar $\mathrm{P}$, Chandrashekhara $\mathrm{SH}$, Kumar A: Unravelling chloroma: review of imaging findings. Br J Radiol 2017, 90:20160710.

3. Byrd JC, Weiss RB, Arthur DC, Lawrence D, Baer MR, Davey F, Trikha ES, Carroll AJ, Tantravahi R, Qumsiyeh M, Patil SR, Moore JO, Mayer RJ, Schiffer CA, Bloomfield CD: Extramedullary leukemia adversely affects hematologic complete remission rate and overall survival in patients with $t(8 ; 21)$ (q22;q22): results from Cancer and Leukemia Group B 8461. J Clin Oncol 1997, 15:466-475.

4. Almond LM, Charalampakis M, Ford SJ, Gourevitch D, Desai A: Myeloid Sarcoma: Presentation, Diagnosis, and Treatment. Clin Lymphoma Myeloma Leuk 2017, 17:263-267.

5. Bakst RL, Dabaja BS, Specht LK, Yahalom J: Use of Radiation in Extramedullary Leukemia/Chloroma: Guidelines From the International Lymphoma Radiation Oncology Group. Int J Radiat Oncol Biol Phys 2018, 102:314-319.

6. Bakst R, Wolden S, Yahalom J: Radiation therapy for chloroma (granulocytic sarcoma). Int J Radiat Oncol Biol Phys 2012, 82:1816-1822.

7. Song JH, Son SH, Lee JH, Chung SM, Jang HS, Choi BO: Defining the optimal dose of radiation in leukemic patients with extramedullary lesions. BMC Cancer 2011, 11:428.

8. Lee EY, Anthony MP, Leung AY, Loong F, Khong PL: Utility of FDG PET/CT in the assessment of myeloid sarcoma. AJR Am J Roentgenol 2012, 198:1175-1179.

9. Young H, Baum R, Cremerius U, Herholz K, Hoekstra O, Lammertsma AA, Pruim J, Price P: Measurement of clinical and subclinical tumour response using [18F]-fluorodeoxyglucose and positron emission tomography: review and 1999 EORTC recommendations. European Organization for Research and Treatment of Cancer (EORTC) PET Study Group. Eur J Cancer 1999, 35:1773-1782.

10. Kaur V, Swami A, Alapat D, Abdallah AO, Motwani P, Hutchins LF, Jethava Y: Clinical characteristics, molecular profile and outcomes of myeloid sarcoma: a single institution experience over 13 years. Hematology 2018, 23:17-24.

11. Kang KM, Choi BO, Chai GY, Kang YN, Jang HS, Kim HJ, Min WS, Kim CC, Choi Ihl Bohng: Effect of cytarabine, melphalan, and total body irradiation as conditioning for autologous stem cell transplantation for patients with AML in first remission. Radiat Oncol J 2003, 21:192-198.

12. Movassaghian M, Brunner AM, Blonquist TM, Sadrzadeh H, Bhatia A, Perry AM, Attar EC, Amrein PC, Ballen KK, Neuberg DS, Fathi AT: Presentation and outcomes among patients with isolated myeloid sarcoma: a Surveillance, Epidemiology, and End Results database analysis. Leuk Lymphoma 2015, 56:1698-1703.

13. Lan T-Y, Lin D-T, Tien H-F, Yang R-S, Chen C-Y, Wu KJAh: Prognostic factors of treatment outcomes in patients with granulocytic sarcoma. Acta Haematol 2009, 122:238-246. 
14. Stölzel F, Lüer T, Löck S, Parmentier S, Kuithan F, Kramer M, Alakel NS, Sockel K, Taube F, Middeke JM, Schetelig J, Röllig C, Paulus T, Kotzerke J, Ehninger G, Bornhäuser M, Schaich M, Zoephel K: The prevalence of extramedullary acute myeloid leukemia detected by (18)FDG-PET/CT: final results from the prospective PETAML trial. Haematologica 2020, 105:1552-1558.

\section{Figures}

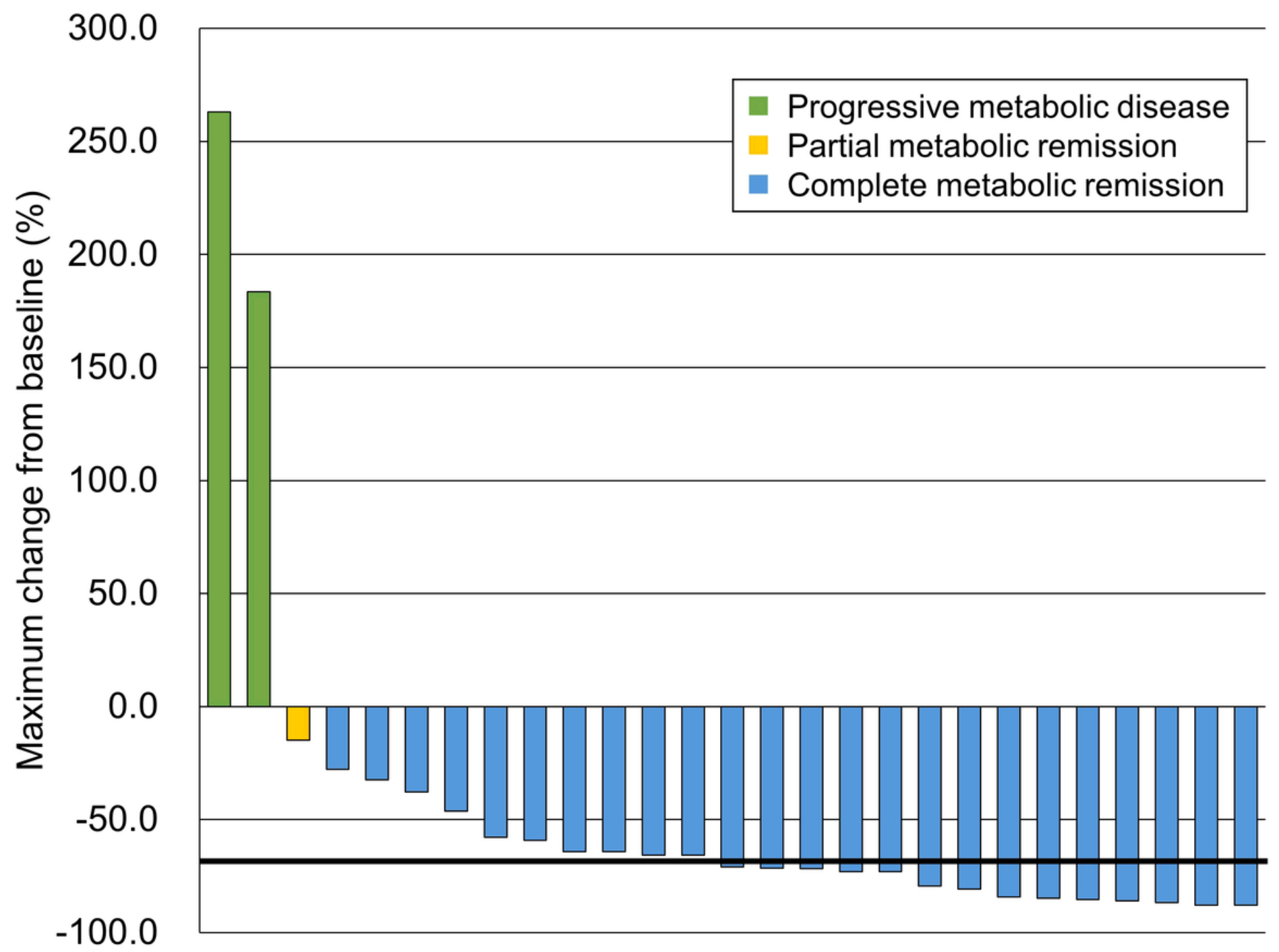

Figure 1

Waterfall plot of the maximum change in SUVmax. SUVmax, maximum standardized uptake value. 


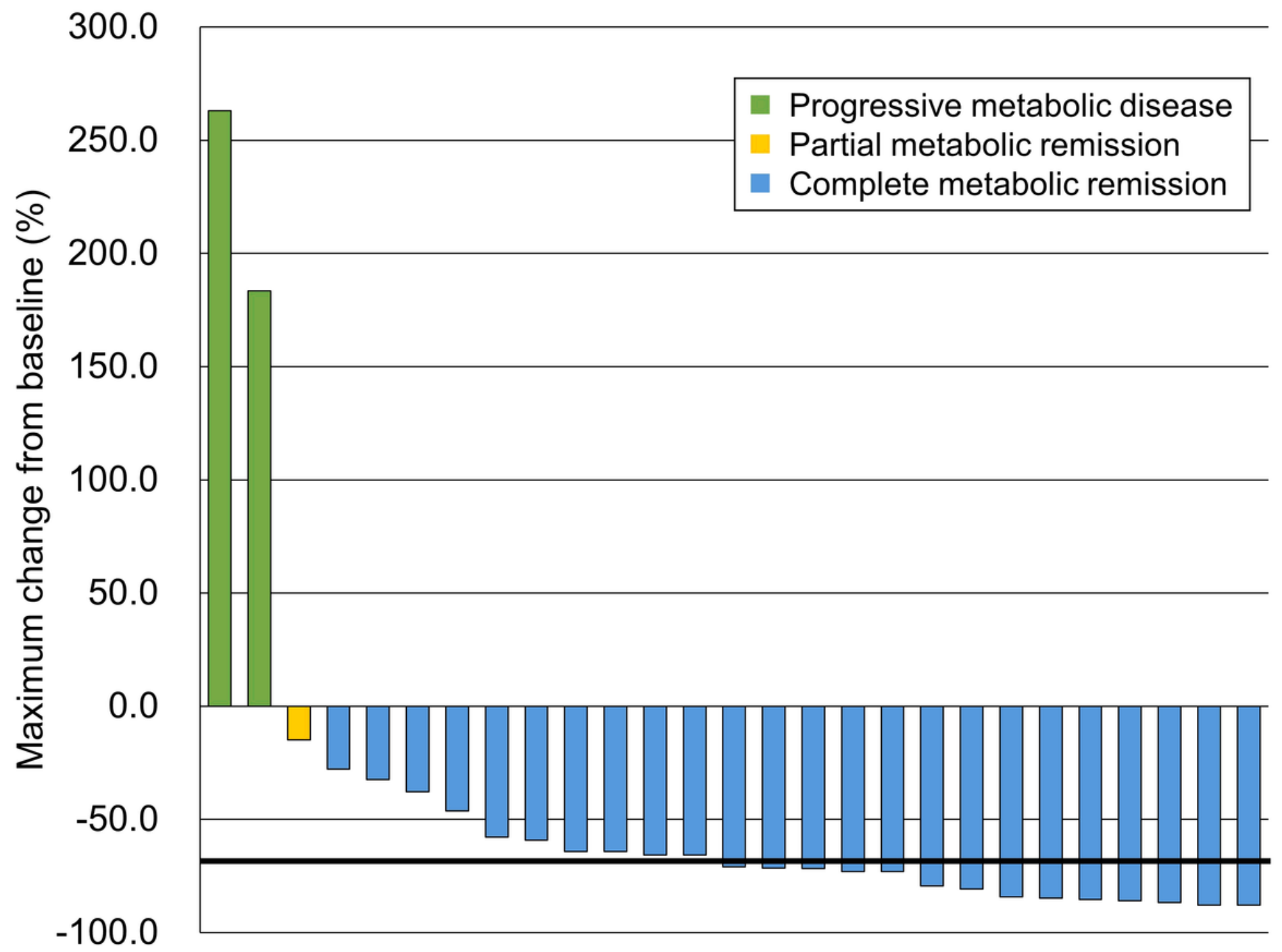

Figure 1

Waterfall plot of the maximum change in SUVmax. SUVmax, maximum standardized uptake value. 


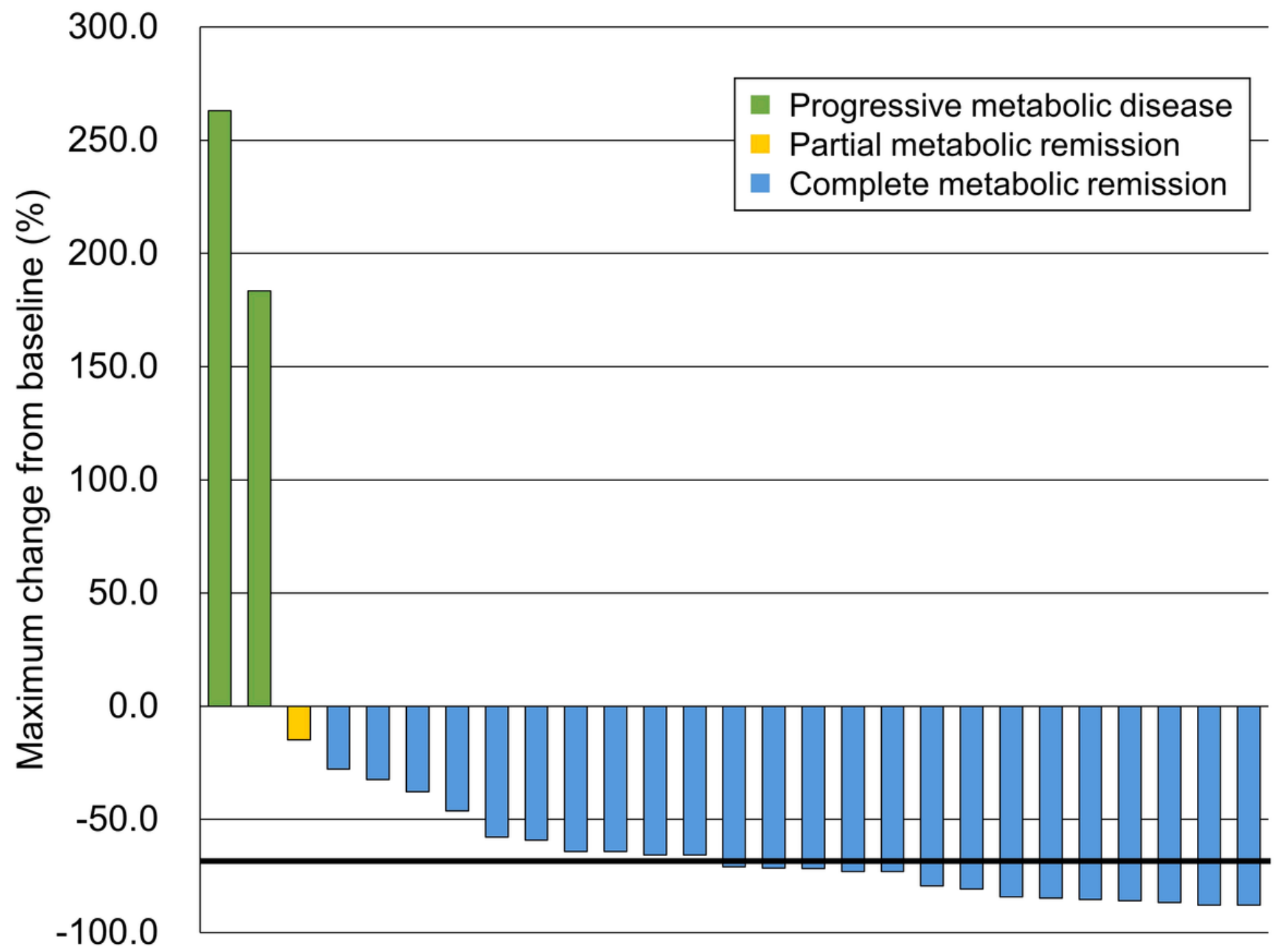

Figure 1

Waterfall plot of the maximum change in SUVmax. SUVmax, maximum standardized uptake value. 


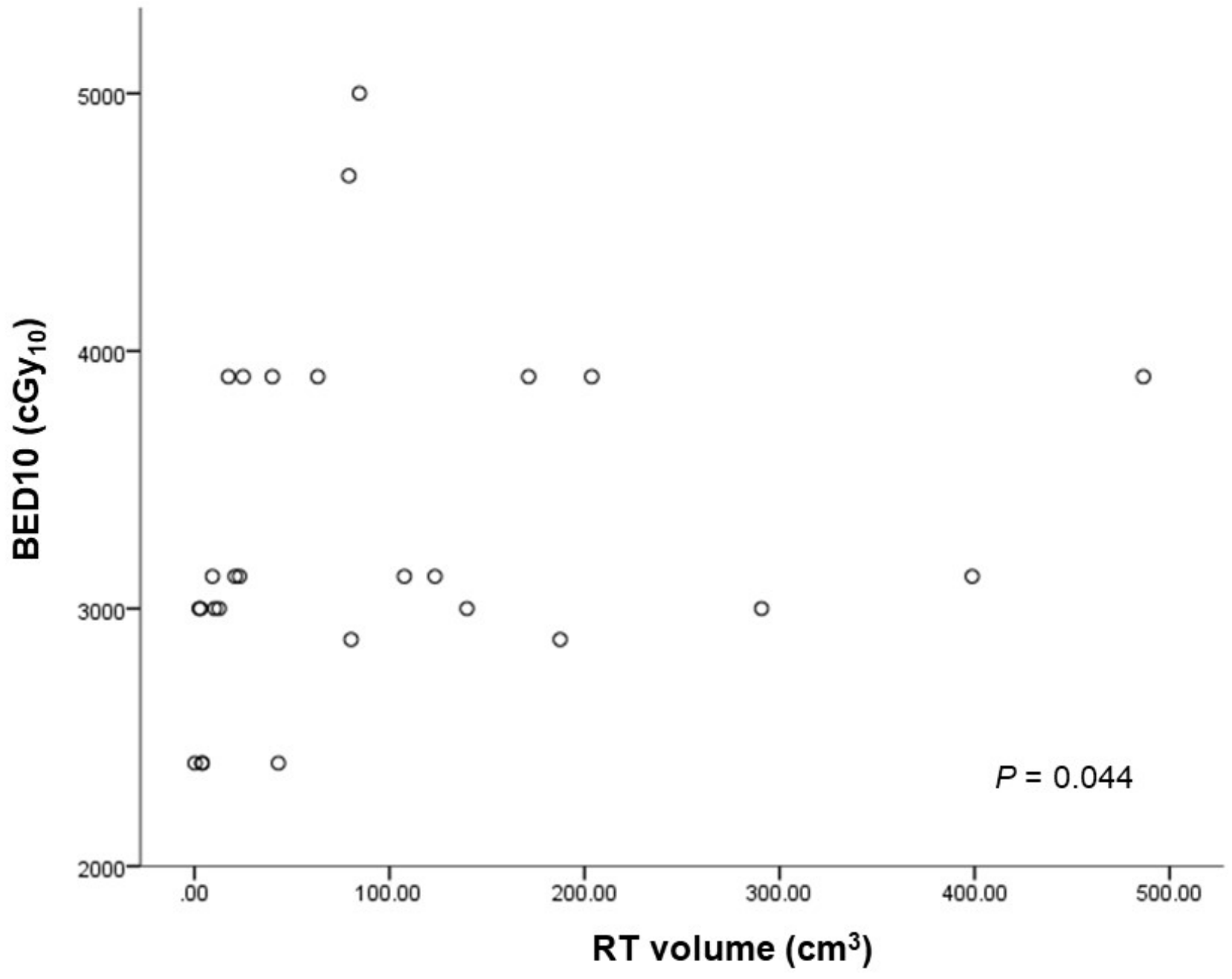

Figure 2

Scatter plot of the linear regression analysis of BED10 and RT volume. BED10, biologically effective dose (alpha/beta ratio $=10) ; \mathrm{RT}$, radiotherapy. 


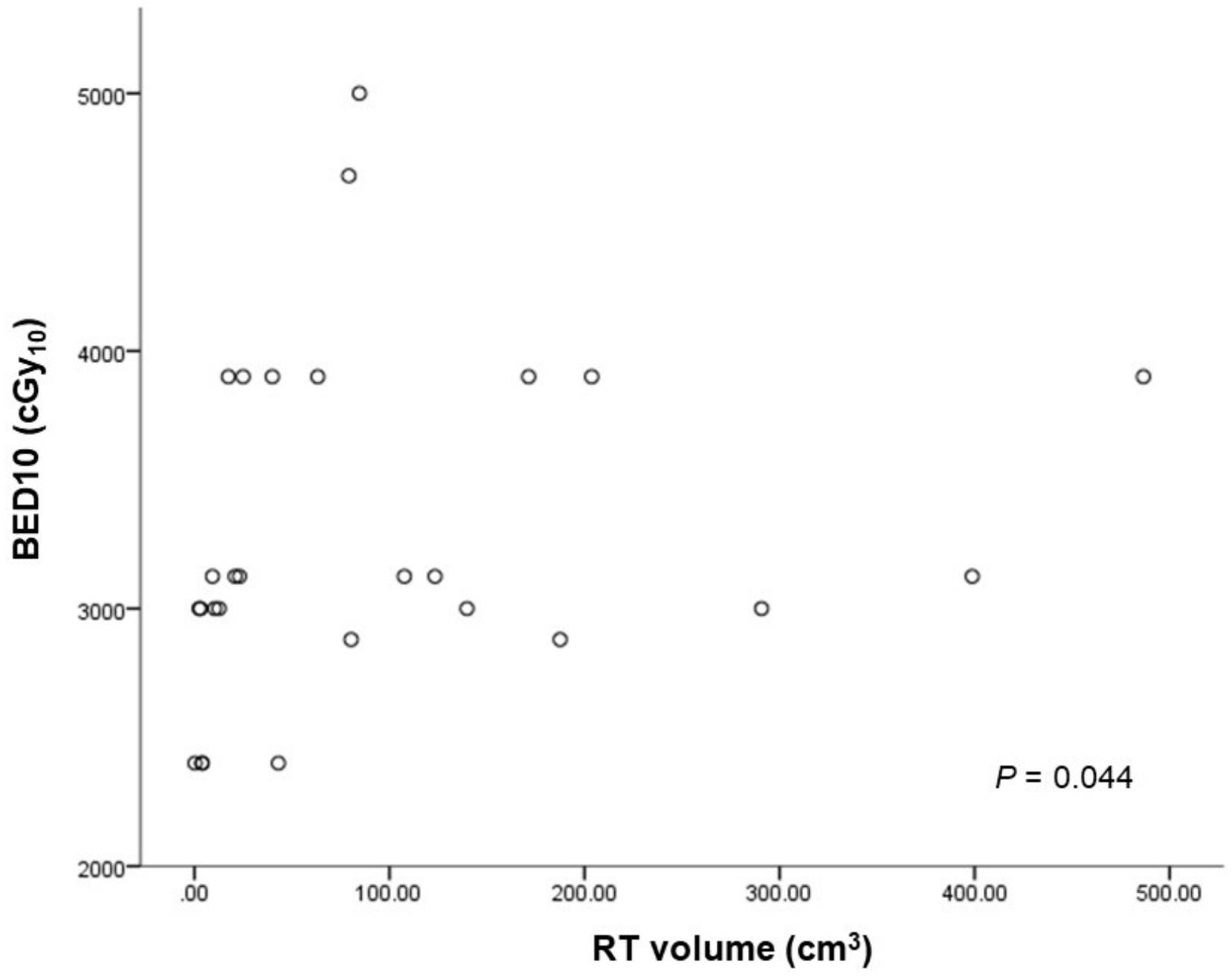

Figure 2

Scatter plot of the linear regression analysis of BED10 and RT volume. BED10, biologically effective dose (alpha/beta ratio $=10) ; \mathrm{RT}$, radiotherapy. 


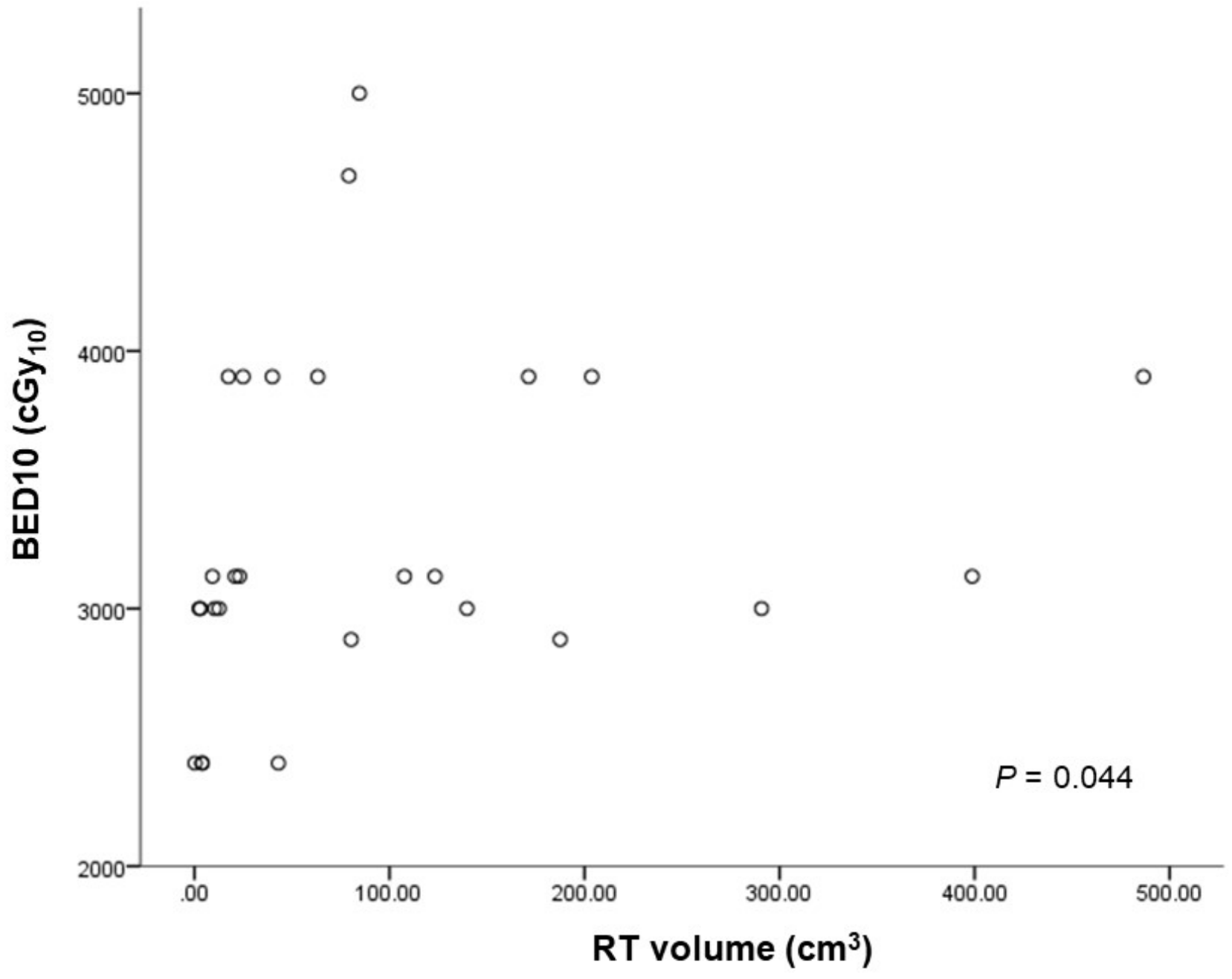

Figure 2

Scatter plot of the linear regression analysis of BED10 and RT volume. BED10, biologically effective dose (alpha/beta ratio $=10) ; \mathrm{RT}$, radiotherapy. 